COMPOSITION AND ORIGIN OF COMETARY MATERIALS

Cover illustration: Picture of Come Hale-Bopp taken on March 21, 1997 with the Schmidt 92/67 telescope by Giovanni Costa, Sergio Dalle Ave and Claudio Lissandrini, Osservatorio Astronomico di Padova, Sede di Asiago. 
Volume 8

The International Space Science Institute is organized as a foundation under Swiss law. It is funded through recurrent contributions from the European Space Agency, the Swiss Confederation, the Swiss National Science Foundation, and the Canton of Bern. For more information, see the homepage at http://www.issi.unibe.ch/ 


\title{
COMPOSITION AND ORIGIN OF COMETARY MATERIALS
}

\author{
Proceedings of an ISSI Workshop, \\ 14-18 September 1998, Bern, Switzerland
}

\author{
Edited by \\ K. ALTWEGG \\ Physikalisches Institut, Universität Bern, CH-3012 Bern, Switzerland \\ P. EHRENFREUND \\ Leiden Observatory, 2300 RA Leiden, The Netherlands \\ J. GEISS \\ International Space Science Institute, CH-3012, Bern, Switzerland \\ W. F. HUEBNER \\ Southwest Research Institute, San Antonio, TX 78228-0510, USA
}

Reprinted from Space Science Reviews, Volume 90, Nos. 1-2, 1999

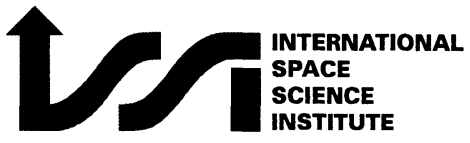

SPRINGER-SCIENCE+BUSINESS MEDIA, B.V. Space Sciences Series of ISSI 
A C.I.P. Catalogue record for this book is available from the Library of Congress.

ISBN 978-94-010-5830-8 ISBN 978-94-011-4211-3 (eBook)

DOI 10.1007/978-94-011-4211-3

Printed on acid-free paper

All Rights Reserved

(C) 1999 Springer Science+Business Media Dordrecht

Originally published by Kluwer Academic Publishers in 1999

No part of the material protected by this copyright notice may be reproduced or utilized in any form or by any means, electronic or mechanical, including photocopying, recording or by any information storage and retrieval system, without written permission from the copyright owner. 


\section{TABLE OF CONTENTS}

Foreword

\section{I: GASES AND GRAINS IN THE COMA}

K. ALTWEGG, H. BALSIGER and J. GEISS / Composition of the Volatile Material in Halley's Coma from In Situ Measurements

J. CROVISIER and D. BOCKELÉE-MORVAN / Remote Observations of the Composition of Cometary Volatiles

R. MEIER and T. OWEN / Cometary Deuterium

P. EBERHARDT / Comet Halley's Gas Composition and Extended Sources: Results from the Neutral Mass Spectrometer on Giotto

M.C. FESTOU / On the Existence of Distributed Sources in Comet Comae

A. LI and J.M. GREENBERG / The Distributed CO in Comet Halley

E. KÜHRT / $\mathrm{H}_{2} \mathrm{O}$ Activity of Comet Hale-Bopp

G. CREMONESE / Hale-Bopp and Its Sodium Tails

E.K. JESSBERGER / Rocky Cometary Particulates: Their Elemental, Isotopic and Mineralogical Ingredients

M.S. HANNER / The Silicate Material in Comets

M.N. FOMENKOVA/ On the Organic Refractory Component of Cometary Dust

\section{II: FROM COMA ABUNDANCES TO NUCLEUS COMPOSITION}

W.F. HUEBNER and J. BENKHOFF / From Coma Abundances to Nucleus Composition

A. ENZIAN / On the Prediction of CO Outgassing from Comets HaleBopp and Wirtanen

J. BENKHOFF / On the Flux of Water and Minor Volatiles from the Surface of Comet Nuclei

J.M. GREENBERG and A. LI / Morphological Structure and Chemical Composition of Cometary Nuclei and Dust 
A.C. LEVASSEUR-REGOURD / Polarization of Light Scattered by Cometary Dust Particles: Observations and Tentative Interpretations

D. PRIALNIK and M. PODOLAK / Changes in the Structure of Comet Nuclei due to Radioactive Heating

\section{III: ORIGIN OF COMETARY MATERIALS}

G. WINNEWISSER and C. KRAMER / Spectroscopy Between the Stars

W.M. IRVINE / The Composition of Interstellar Molecular Clouds

L.J. ALLAMANDOLA, M.P. BERNSTEIN, S.A. SANDFORD and R.L. WALKER / Evolution of Interstellar Ices

P. EHRENFREUND / An ISO View on Interstellar and Cometary Ice Chemistry

B. FEGLEY Jr. / Chemical and Physical Processing of Presolar Materials in the Solar Nebula and the Implications for Preservation of Presolar Materials in Comets

J. GEISS, K. ALTWEGG, H. BALSIGER and S. GRAF / Rare Atoms, Molecules and Radicals in the Coma of P/Halley

G. STRAZZULLA / Ion Irradiation and the Origin of Cometary Materials 269-274

J.F. KERRIDGE / Formation and Processing of Organics in the Early Solar System

K. LODDERS and R. OSBORNE/ Perspectives on the Comet-AsteroidMeteorite Link

\section{IV: CRITICAL MEASUREMENTS FOR THE FUTURE}

P.R. WEISSMAN / Diversity of Comets: Formation Zones and Dynamical Paths

G. SCHWEHM and R. SCHULZ / Rosetta Goes to Comet Wirtanen

R. SCHULZ and G. SCHWEHM / Coma Composition and Evolution of Rosetta Target Comet 46P/Wirtanen

W.T. HUNTRESS Jr. / Missions to Comets and Asteroids

L. COLANGELI, V. MENNELLA, J.R. BRUCATO, P. PALUMBO and A. ROTUNDI / Characterization of Cosmic Materials in the Laboratory 
S.A. STERN / Studies of Comets in the Ultraviolet: The Past and the Future

A.J. BALL, H.U. KELLER and R. SCHULZ / Critical Questions and Future Measurements - Collated Views of the Workshop Participants

\section{V: SUMMARY AND INDEXES}

K. ALTWEGG, P. EHRENFREUND, J. GEISS, W.F. HUEBNER and A.C. LEVASSEUR-REGOURD / Cometary Materials: Progress Toward Understanding the Composition in the Outer Solar Nebula

Subject Index

Comet Index 398

Molecule Indexes

Abbreviations 408

Author Index

List of Participants 
Composition and Origin of Cometary Materials

ISSI Workshop, September 14 - 18, 1998, Bern, Switzerland

\section{Group Photographs}
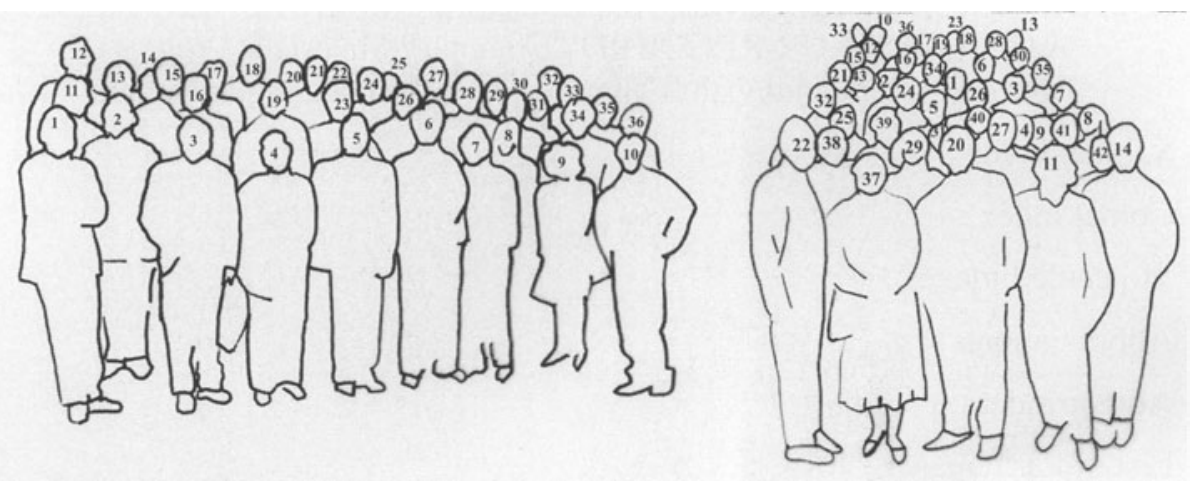

1. J.F. Kerridge

2. G. Strazzulla

3. H. Rickman

4. S.A. Stern

5. P. Eberhardt

6. W.T. Huntress Jr.

7. J. Geiss

8. S. Wenger

9. D. Prialnik

10. R. Meier

11. J. Crovisier

12. E.K. Jessberger

13. W. Benz

14. S. Verani

15. L. Colangeli
16. B. Fegley Jr.

17. K. Lodders

18. H.U. Keller

19. G. Cremonese

20. M.J. Mumma

21. W.F. Huebner

22. L.J. Allamandola

23. C. Barbieri

24. J.F. Crifo

25. P. Ehrenfreund

26. P.R. Weissman

27. A. Enzian

28. A.J. Ball

29. R. Schulz

30. S. Graf
31. L.M. Lara

32. J. Benkhoff

33. E. Kührt

34. G. Winnewisser

35. J.M. Greenberg

36. K. Altwegg

37. M.S. Hanner

38. W.M. Irvine

39. M.C. Festou

40. U. Pfander

41. M.N. Fomenkova

42. G. Nusser Jiang

43. A.C. Levasseur-Regourd 

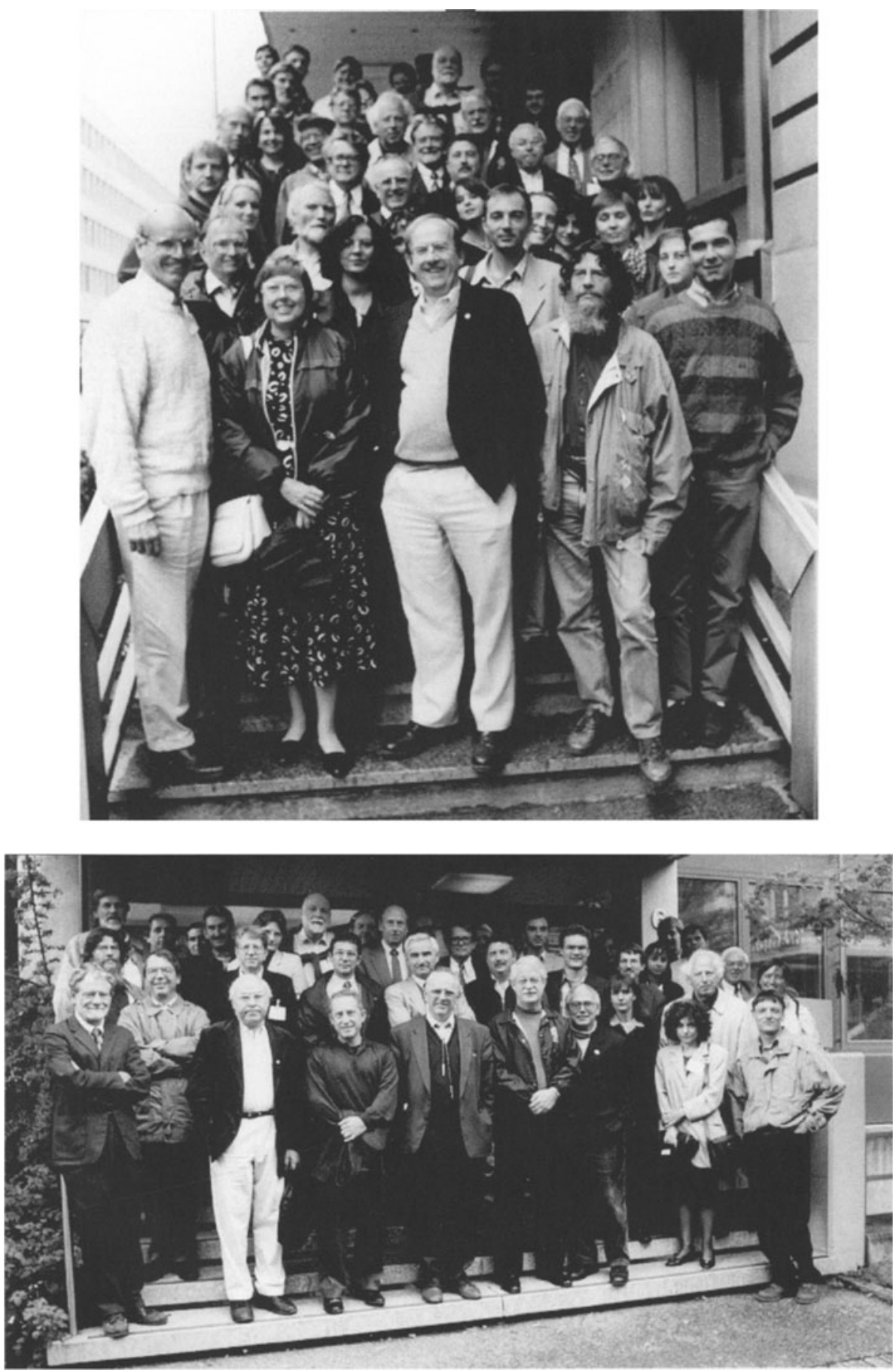


\section{FOREWORD}

The current volume, the eighth in the "Space Sciences Series of ISSI" (International Space Science Institute) presents the proceedings of the workshop on "Composition and Origin of Cometary Materials", which was held at ISSI in Bern on September 14-18, 1998.

Following the advice of three ISSI Working Groups that had studied several aspects of comet research, ISSI appointed four convenors, Kathrin Altwegg (University of Bern), Pascale Ehrenfreund (University of Leiden), Johannes Geiss (ISSI), and Hans Rickman (Astronomical Observatory Uppsala), to set up the programme and to nominate participants.

The topic of this workshop is highly interdisciplinary in nature, and consequently scientists from different fields, including optical and radio astronomers, chemists, experimental physicists, as well as theorists and modellers of cometary and interstellar processes, participated.

The data discussed at the workshop were mainly those obtained in 1986 of the coma of Comet Halley by the Giotto spacecraft of ESA and the Vega spacecraft of the Soviet Union and those more recently obtained by remote sensing of the comae of the bright Comets Hyakutake (C/1996 B2) and Hale-Bopp (C/1995 O1). In addition, new laboratory data and model results were presented and discussed.

Comparison of data on interstellar and cometary grains confirmed earlier ideas on their origin and evolution: Tiny refractory grains are produced in stellar envelopes and explosions and transported into the interstellar medium. When a dense Interstellar Molecular Cloud (IMC) forms, these grains serve as nuclei for the condensation of gas-phase molecules and radicals. During recycling of such cloud materials through the diffuse interstellar medium, the mantles of the grains are processed by UV and cosmic radiation. It is thought that the organic material (also called CHON) found in the coma of Halley's Comet is the result of such processing. Cycling between IMCs and the diffuse interstellar medium may have occurred several times until $4.6 \times 10^{9}$ years ago. At that time a molecular cloud collapsed to form protostellar discs - among them the solar nebula. During the collapse phase and in the solar nebula some evaporation, molecular alteration, and recondensation occurred, but it appears that the CHON material found in comets has preserved most of its interstellar identity.

New results presented at the workshop confirmed that at least some constituents of comet ices are also of interstellar origin: Two types of species are considered as specific indicators for IMC chemistry: deuterated molecules and certain radicals. In IMCs, deuterium is highly enriched in condensable molecules. Similarly, D is enriched (above the proto-solar abundance) by an order of magnitude in cometary 
water and by two orders of magnitude in the hydrogen-cyanide of Comet HaleBopp. Such strong enrichments result from ion-molecule reactions at very low temperatures. The $\mathrm{D} / \mathrm{H}$ ratios measured in cometary water and cometary hydrogen are similar to values found in the hot cores of IMCs. This suggests that significant fractions of $\mathrm{H}_{2} \mathrm{O}$ and $\mathrm{HCN}$ molecules of Comets Halley and Hale-Bopp were synthesised in the ancient IMC from which the solar system formed $4.6 \times 10^{9}$ years ago. Some $\mathrm{CH}_{2}$ and $\mathrm{C}_{4} \mathrm{H}$, which were detected in the inner coma of Comet Halley, appear to be primary species that have been vaporised from the ice of the nucleus. If so, these radicals are likely to be of IMC origin, and their survival places important constraints on the thermal history of comet ices.

This volume is a collection of the papers resulting from the invited and contributed presentations, each of which was reviewed by an independent referee. Furthermore, it contains an article by the four editors and Ann-Chantal LevasseurRegourd that summarises and integrates the various views presented at the workshop. The article concludes with a list of recommendations. At the end, the reader will find a list of abbreviations as well as a subject index and an index of comets. Furthermore, we have included an index of molecules, put together by Stephan Graf and Ursula Pfander, which should be useful for the reader. Comet missions mentioned in this volume are those that had been approved at the time of the workshop. This list may change even before this volume is published.

We wish to express our sincere thanks to all those who have made this volume possible. First of all, we should like to thank the authors for their contributions and the reviewers for their critical and timely reports, which have significantly contributed to the quality of this volume. It is a pleasure to thank the directorate and the staff of ISSI for their support in organising this workshop, in particular D. Taylor, X. Schneider, G. Nusser Jiang, U. Pfander, and S. Wenger. We thank Stephan Graf for designing the chemical index and his help with the manuscript, Paul Wild for his advice concerning the Comet Index, and Diane Taylor for correcting the English in some of the papers. The editors could not have published this volume at the foreseen date without the support of the editorial assistant Ursula Pfander. Her help to authors and editors in producing camera-ready texts, tables, and figures, and her preparing the camera-ready version of the volume including the indexes is very much appreciated.

September 1999

K. Altwegg, P. Ehrenfreund, J. Geiss, W. F. Huebner 